\title{
Effects of laser parameters and scanning strategy on structural and mechani- cal properties of 3D NiTi implants fabricated with selective laser melting
}

\author{
de Wild $\mathrm{M}^{1}$, Schollbach $\mathrm{T}^{1}$, Schumacher $\mathrm{R}^{1}$, Schkommodau $\mathrm{E}^{1}$, Bormann $\mathrm{T}^{1}$ \\ ${ }^{1}$ Institute for Medical and Analytical Technologies, School of Life Sciences, University of Applied Sciences \\ Northwestern Switzerland, 4032 Muttenz, Switzerland
}

Michael.deWild@fhnw.ch

\begin{abstract}
:
The additive manufacturing method Selective Laser Melting (SLM) allows the creation of open-porous shape memory structure directly from CAD data. However, during the SLM process, laser parameters and scanning strategy must be carefully selected. In this study, we show that they have a significant effect on geometrical and mechanical properties of NiTi scaffolds for the treatment of large bone defects.
\end{abstract}

Keywords: NiTi, shape memory alloy, SLM, scanning strategy, laser parameters

\section{Introduction}

Implant morphology, biocompatibility and mechanical properties are strongly influenced by the SLM manufacturing process. This process is controlled by a variety of parameters which are stored in a material file: scanning strategy, laser energy, exposure time, point distance, hatch distance, minimal hatch length, fill contour offset, lens position, energy density, etc. The geometry, mechanical property and microscopic structure strongly depend on these settings [1]. The $\mu \mathrm{CT}$ and mechanical investigation give insight into the structural characteristics of the SLM processed scaffolds [2].

\section{Methods}

The NiTi scaffolds with a rhombo-dodecahedral unit cell and strut thicknesses around $300 \mu \mathrm{m}$ (see fig. 1A) are produced with a Realizer 100 system (Borchen, Germany) equipped with a $100 \mathrm{~W}$ fiber laser [3]. The compressive strength was measured by a Zwick/Roell Z100 testing machine. The mass of the open-porous scaffolds was determined gravimetrically (Acculab). The materials porosity, defined as closed micro-pores within the metallic struts, was determined by analysing the 3D dataset obtained by a SkyScan $1172 \mu \mathrm{CT}$ system with the CTAn software (SkyScan NV, Belgium), see fig. 1B.

\section{Results}

The scaffolds mass and therefore the overall porosity are found in the range of $0.145-0.366 \mathrm{~g}$, resp. $71.6-88.7 \%$ when applying different laser parameters and scanning strategies. As expected, the compressive strength of the scaffolds is highly correlated to the structures mass, see fig $2 \mathrm{~A}$. The tomographic analyses reveal slightly more micro-pores in heavier scaffolds, see fig. $2 \mathrm{~B}$.

\section{Discussion}

The SLM process allows the creation of open-porous NiTi scaffolds with porosity up to $88 \%$. The lattice material itself is very dense (density $>99.8 \%$ ). The laser parameter and scanning strategy have to be considered as they affect the geometry and mechanical properties. These parameters were identified in order to improve the implant design and fabrication.
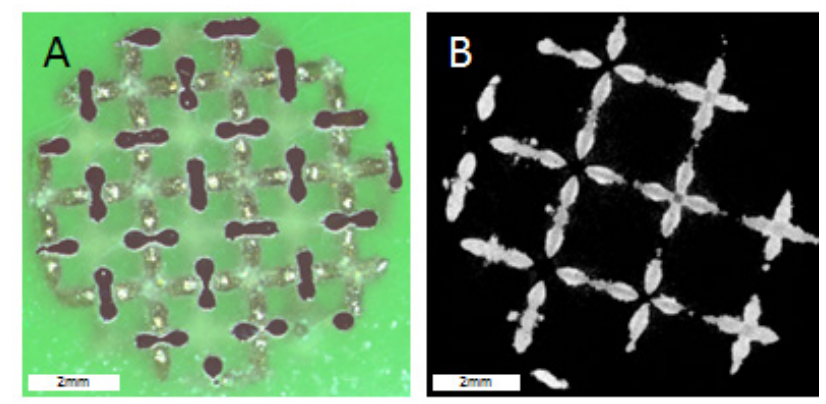

Figure 1: A) metallographic section of the NiTi scaffold. B) Cross section of reconstructed $\mu \mathrm{CT}$ dataset.
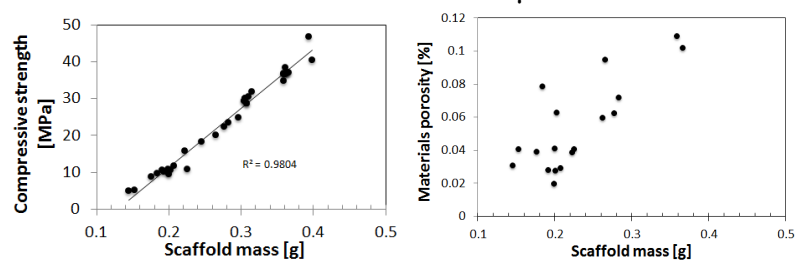

Figure 2: Correlation between the scaffold mass and A) the mechanical strength, B) the materials porosity.

\section{Acknowledgement}

The multi-disciplinary team gratefully acknowledges the financial support of the Swiss National Science Foundation within the program NRP 62 'Smart Materials'.

\section{Bibliography}

[1] Stamp, R. et. al.: The development of a scanning strategy for the manufacture of porous biomaterials by selective laser melting, J Mater Sci Mater Med, vol. 20, pp. 1839-1848, September 2009.

[2] Lin, C.Y. et. al.: Structural and mechanical evaluations of a topology optimized titanium interbody fusion cage fabricated by selective laser melting process, $J$ Biomed Mater Res A, vol. 88, pp. 272-279, November 2007.

[3] Bormann, T., et al.: Tailoring Selective Laser Melting Process Parameters for NiTi Implants, J Mat Eng Perf, vol. 21, pp. 2519-2514, July 2012. 